\title{
Circuito equivalente de un transformador con regulación
}

\author{
Equivalent circuit of a regulating transformer
}

\author{
Juan A. Martínez-Velasco ${ }^{1} \quad$ Francisco de León ${ }^{2}$ \\ Recibido 16 de junio de 2010, aceptado 23 de noviembre de 2010 \\ Received: June 16, 2010 Accepted: November 23, 2010
}

\begin{abstract}
RESUMEN
Este artículo presenta una metodología para la obtención y utilización del circuito equivalente de un transformador con regulación en el que es posible variar su relación de transformación. Este aspecto está insuficientemente tratado en la mayoría de libros de texto actuales en los que el circuito de un transformador con regulación se presenta de forma poco justificada. Este documento muestra cómo obtener el circuito equivalente del transformador y cómo aplicarlo utilizando tanto valores reales como valores por unidad (pu). El tratamiento con valores por unidad se puede realizar seleccionando los valores base de forma arbitraria, lo que supone una ventaja evidente cuando se trata de determinar alguna de las tensiones nominales del transformador en estudio. Aunque el objetivo del artículo es el de presentar un método para transformadores con regulación, el método es también válido para transformadores con relación de transformación constante en los que se tenga que determinar alguna de sus tensiones nominales. El artículo incluye dos ejemplos con campos de aplicación muy distintos que servirán para ilustrar las ventajas del método propuesto.
\end{abstract}

Palabras clave: Transformador de potencia, transformador con tomas, circuito equivalente, análisis en régimen permanente, modelación, valores por unidad.

\begin{abstract}
This paper presents a methodology aimed at obtaining and applying the equivalent circuit of a regulating transformer, whose turn ratio can be varied. This aspect is insufficiently treated in most current textbooks in which the equivalent circuit of a tapped transformer is not well justified. This document shows how to obtain the equivalent circuit of the transformer and how to apply it using either physical quantities or per unit (pu) quantities. The usage of per unit values can be performed by selecting the base quantities in an arbitrary manner, which is an obvious advantage when one of the rated voltages must be estimated. Although the goal of the paper is to develop a method for regulating transformers (e.g., under load tap changers, ULTC), it can be applied to transformers with a fixed turn ratio for which one of the rated voltages is unknown. The paper includes two illustrative examples with different fields of application.
\end{abstract}

Keywords: Power transformer, tap changer, equivalent circuit, steady-state analysis, modeling, per unit values.

\section{INTRODUCCIÓN}

El transformador con tomas es un dispositivo muy común en las redes de transporte y distribución de energía eléctrica, incluso en instalaciones eléctricas de alta tensión. Se trata de un dispositivo muy útil para el control de tensiones [1-5]. Si además de variar su relación de transformación, también se

\footnotetext{
1 Departament d'Enginyería Elèctrica. Universitat Politècnica de Catalunya. Av. Diagonal 647, CP 08028. Barcelona, España. E-mail: martinez@ee.upc.edu

2 Department of Electrical and Computer Engineering. Polytechnic Institute of NYU. Brooklyn, NY 11201. USA. E-mail: fdeleon@poly.edu
} 
puede variar el desfase entre las tensiones en sus terminales, entonces también es un dispositivo muy útil para el control de flujos de potencia [6-7].

Uno de los problemas que se presenta cuando se trata de realizar cálculos con este tipo de transformador es el tratamiento de su circuito equivalente. El problema se complica cuando se pretende realizar cálculos con cantidades por unidad (pu), donde los valores base y los valores nominales no coinciden.

El cálculo de los parámetros del circuito equivalente de un transformador en valores por unidad es muy simple cuando los valores base seleccionados y los valores nominales del transformador coinciden. Sin embargo, existen varias razones por las que no es posible seleccionar los valores base iguales a los valores nominales; por ejemplo, cuando el problema consiste en determinar la toma o la tensión nominal (de primario o secundario) que se debe asignar a un transformador para conseguir una determinada tensión en cierto nudo de la red en estudio, o cuando el sistema tiene varios transformadores y no es posible seleccionar los valores base iguales a los valores nominales de todos ellos. Por otra parte, existen muy pocos libros de texto que presenten de forma metódica la obtención del circuito equivalente, y que justifiquen tanto la selección de valores base como las hipótesis de cálculo empleadas.

El objetivo de este artículo es mostrar cómo se pueden obtener y aplicar los circuitos equivalentes de un transformador de dos arrollamientos con relación de transformación variable, cuyos parámetros pueden estar expresados en cantidades físicas o en valores por unidad, si los valores base se eligen de forma arbitraria.

El circuito equivalente de un transformador depende del tipo de estudio a realizar y sobre todo del rango de frecuencias que pueden aparecer en las ondas de tensión y corriente del sistema en estudio. Para un análisis detallado de los modelos de transformador a emplear, el lector interesado puede consultar las referencias [8-12]. Los circuitos equivalentes estudiados en este artículo sólo son válidos para el análisis de redes en régimen permanente y equilibrado; por ejemplo, para estudios de flujos de carga (load flow) en los que se utilizan modelos monofásicos.
El documento ha sido organizado como sigue. Inicialmente presenta una breve introducción de los circuitos equivalentes de un transformador monofásico de dos arrollamientos en régimen permanente, así como de los circuitos equivalentes empleados en este trabajo. A continuación de justifica la deducción de los circuitos equivalentes de un transformador con tomas y con parámetros expresados en cantidades físicas o en valores por unidad. La aplicación de estos circuitos se ilustra mediante dos ejemplos relacionados con aplicaciones muy distintas: la selección de tensión nominal y el cálculo de las corrientes de cortocircuito permanentes en caso de falta simétrica.

\section{CIRCUITOS EQUIVALENTES DE UN TRANSFORMADOR}

La Figura 1 muestra el circuito equivalente de un transformador de dos arrollamientos en el que sus lados primario y secundario están relacionados mediante la relación de transformación $N_{p} / N_{s}$. Este circuito puede servir para representar un transformador monofásico o cualquiera de las fases de un transformador trifásico, tanto en régimen permanente como en procesos transitorios de baja frecuencia. En caso de tener que analizar el comportamiento completo de un transformador trifásico, además de incluir la representación de las tres fases, sería necesario tener en cuenta el tipo de conexión en ambos lados del transformador.

En este trabajo sólo se analizan circuitos adecuados para representar un transformador de dos arrollamientos funcionando en régimen permanente y en condiciones de carga simétrica y equilibrada. En tales condiciones, un transformador trifásico se puede representar mediante un circuito equivalente monofásico.

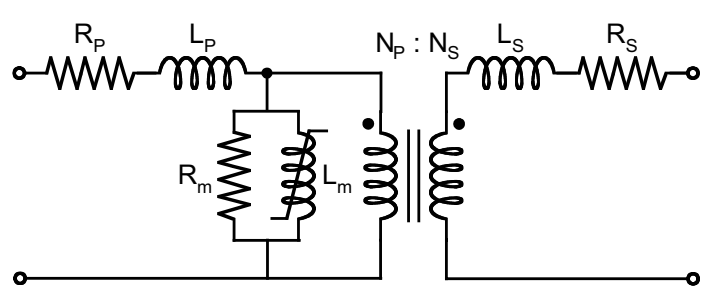

Figura 1. Circuito equivalente de un transformador con dos arrollamientos. 
Los parámetros que aparecen en el circuito de la Figura 1 tienen un significado físico muy concreto:

- $\quad R_{m}$ y $L_{m}$ son los parámetros del núcleo del transformador; $R_{m}$ representa las pérdidas en el núcleo, mientras que $L_{m}$ representa el flujo de magnetización confinado en el núcleo y común a los dos arrollamientos del transformador.

- $\quad R_{p}, L_{p}, R_{s}$ y $L_{s}$ son los parámetros de los arrollamientos; $R_{p}$ y $R_{s}$ representan las pérdidas por efecto Joule de los dos arrollamientos del transformador, y $L_{p}$ y $L_{s}$ representan los flujos de dispersión de cada arrollamiento.

- La relación $N_{p} / N_{s}$ es la relación entre el número de espiras de los lados primario y secundario del transformador, o lo que es igual la relación de transformación entre tensiones y corrientes nominales de ambos lados.

La representación de la Figura 1 no es la única utilizada en estudios con transformadores. Es muy corriente el uso de circuitos equivalentes en los que los dos arrollamientos del transformador se representan mediante una sola resistencia y una sola inductancia, ya sea referida al lado primario o al lado secundario. Por otro lado, los parámetros del núcleo, $R_{m}$ y $L_{m}$, también podrían haber sido situados en el lado secundario. En todos los casos se ha supuesto que el parámetro $L_{m}$ es no lineal. Una representación más rigurosa habría considerado que también es no lineal el parámetro $R_{m}$, y habría tenido en cuenta la dependencia de $R_{m}$ con respecto a la frecuencia.

El circuito de la Figura 1 es utilizado en el cálculo de procesos transitorios de baja frecuencia y en determinados estudios en régimen permanente, aunque en este último caso se suele considerar que el parámetro $L_{m}$ tiene un comportamiento lineal.

Para determinados estudios, el circuito equivalente de un transformador se puede simplificar, prescindiendo de los parámetros que representan el núcleo. En este trabajo se supone que cualquiera de los circuitos de la Figura 2 representa el comportamiento de un transformador con suficiente precisión [6-7, 13-14]. De hecho en algún caso se prescindirá incluso del parámetro resistencia. Para obtener los parámetros de los circuitos de la Figura 2 será necesario conocer los valores obtenidos en el ensayo normalizado en cortocircuito.
Si se utilizan los siguientes símbolos:

- Potencia nominal $S_{n}$, en kVA o MVA

- Tensiones nominales $V_{n 1}, V_{n 2}$, en $\mathrm{kV}$

- Tensión de cortocircuito $\varepsilon_{c c}$, (en pu o en \%)

- Pérdidas por efecto Joule en el ensayo en cortocircuito $\quad W_{c c}$, en $\mathrm{kW}$ o MW

el cálculo de los parámetros del circuito equivalente simplificado y referido al secundario podría ser como sigue (Figura 2b):

$$
\begin{gathered}
Z_{2}=\varepsilon_{c c} \frac{V_{n 2}^{2}}{S_{n}} \\
R_{2}=\frac{W_{c c}}{S_{n}} \frac{V_{n 2}^{2}}{S_{n}} \\
X_{2}=\omega L_{2}=\sqrt{Z_{2}^{2}-R_{2}^{2}} \quad(\omega=2 \pi f)
\end{gathered}
$$

donde $Z_{2}$ es la impedancia total de los arrollamientos, referida al lado secundario, y $f$ es la frecuencia de operación del sistema en el que funciona el transformador.

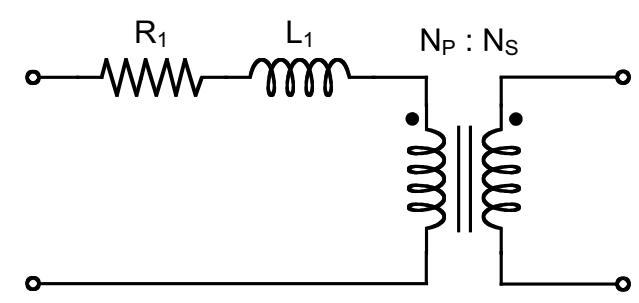

a) Parámetros en el lado primario.

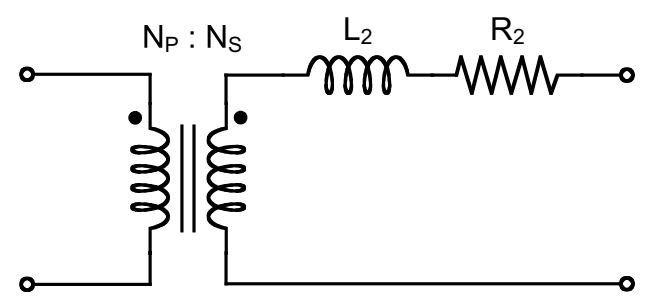

b) Parámetros en el lado secundario.

Figura 2. Circuitos equivalentes simplificados de un transformador con dos arrollamientos.

Por lo que respecta al cálculo de parámetros referidos al lado primario (Figura 2a), sólo es necesario cambiar el subíndice " 2 " por el subíndice " 1 " en el símbolo de tensión nominal y aplicar el mismo proceso. 
Los valores de resistencia, inductancia (o reactancia) e impedancia de los circuitos de la Figura 2 se conocen también como resistencia, inductancia (o reactancia) e impedancia de cortocircuito. Así, por tanto, en el resto del artículo se utilizarán los siguientes símbolos:

$$
\begin{aligned}
& \underline{Z}_{1}=\underline{Z}_{c c 1}=R_{c c 1}+j X_{c c 1} \\
& \underline{Z}_{2}=\underline{Z}_{c c 2}=R_{c c 2}+j X_{c c 2}
\end{aligned}
$$

En la aplicación de estas fórmulas se supone que los valores de $\varepsilon_{c c}$ y $W_{c c}$, son los mismos independientemente de cuál sea el lado por el que se alimenta o el lado por el que se cortocircuita el transformador durante el ensayo en cortocircuito.

\section{CIRCUITOS EQUIVALENTES DE UN TRANSFORMADOR CON REGULACIÓN}

El estudio se realizará con un transformador monofásico del que se conocen la potencia nominal $\left(S_{n}\right)$, las tensiones nominales $\left(V_{n 1}, V_{n 2}\right)$, la tensión de cortocircuito $\left(\varepsilon_{c c}\right)$ y las pérdidas de potencia por efecto Joule obtenidas en el ensayo en cortocircuito $\left(W_{c c}\right)$.

La relación entre los valores nominales de un transformador es:

$$
V_{n 1} I_{n 1}=V_{n 2} I_{n 2}
$$

Los valores base utilizados para obtener el circuito equivalente con parámetros por unidad se escogen inicialmente de forma arbitraria:

$\begin{array}{ll}\text { - Potencia base: } & S_{b} \\ \text { - Tensiones base: } & V_{b 1}, V_{b 2}\end{array}$

De aquí se obtienen las siguientes intensidades e impedancias base:

$$
\begin{gathered}
I_{b 1}=\frac{S_{b}}{V_{b 1}} \quad ; \quad I_{b 2}=\frac{S_{b}}{V_{b 2}} \\
Z_{b 1}=\frac{V_{b 1}^{2}}{S_{b}} \quad ; \quad Z_{b 2}=\frac{V_{b 2}^{2}}{S_{b}}
\end{gathered}
$$

El estudio se realiza suponiendo que ni la tensión aplicada en el ensayo de cortocircuito, $\varepsilon_{c c}$, generalmente expresada en valor porcentual $(\%)$ con respecto a las tensiones nominales, ni las pérdidas de potencia por efecto Joule medidas en el mismo ensayo, $W_{c c}$, dependen de la toma, o sea, de la relación de transformación en el transformador. De forma intuitiva es posible razonar que al cambiar la toma del transformador el flujo de dispersión en el lado de la toma varía de forma proporcional y que, por tanto, la tensión de cortocircuito referida a la nueva tensión nominal seguirá siendo la misma. Por lo que respecta a las pérdidas por efecto Joule, se puede suponer que al variar la toma o la tensión nominal en un lado del transformador, se modifican la resistencia y la intensidad de forma proporcional. Como las pérdidas dependen del cuadrado de la intensidad y la impedancia varía con el cuadrado de la relación de transformación, se puede suponer que ambos efectos se compensan cuando se juntan las pérdidas de los dos arrollamientos en una sola cantidad.

En realidad no es estrictamente correcto suponer que la tensión de cortocircuito de un transformador expresada en forma porcentual es independiente de la toma del transformador. Sin embargo, cuando se trata de obtener el circuito equivalente de un transformador con regulación, esta aproximación es utilizada en los libros de texto y en muchos estudios en los que la representación del transformador se deriva de alguno de los circuitos mostrados en la Figura 2.

La sección ha sido dividida en dos partes, en cada una de las cuales se obtendrá un circuito equivalente del transformador con regulación. Aplicando las hipótesis comentadas en el párrafo anterior, se puede concluir que el valor de la impedancia de cortocircuito de un transformador se puede obtener referida a su lado secundario cuando se conoce la tensión nominal de este lado y las tomas de regulación se sitúan en el lado primario, ver ecuaciones (1). Inversamente, será posible obtener la impedancia de cortocircuito referida al lado primario cuando las tomas de regulación se sitúan en el lado secundario y, por tanto, se conoce la tensión nominal del lado primario. 


\section{Impedancia de cortocircuito vista desde el lado secundario}

La Figura 3 muestra el circuito equivalente de un transformador visto desde su lado secundario, en el que se puede regular su relación de transformación.

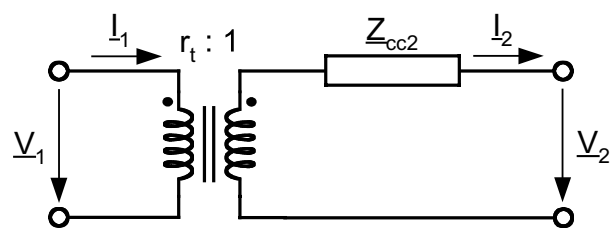

Figura 3. Circuito equivalente de un transformador visto desde el lado secundario.

Las ecuaciones del circuito equivalente se pueden anotar de la siguiente forma:

$$
\begin{aligned}
& \underline{V}_{1}=r_{t}\left(\underline{V}_{2}+\underline{Z}_{c c 2} \underline{I}_{2}\right) \\
& \underline{I}_{1}=\underline{I}_{2} / r_{t}
\end{aligned}
$$

Donde

$$
r_{t}=\frac{V_{n 1}}{V_{n 2}}=\frac{I_{n 2}}{I_{n 1}} \quad ; \quad Z_{c c 2}=\varepsilon_{c c} \frac{V_{n 2}^{2}}{S_{n}}
$$

El valor de $Z_{c c 2}$ se puede calcular cuando se conoce el valor de $V_{n 2}$, tal como muestra la ecuación (6). Los valores de la resistencia, $R_{c c 2}$, y la reactancia de cortocircuito, $X_{c c 2}$, vistas desde el lado secundario, se calculan mediante las ecuaciones (1). En el estudio que se presenta a continuación se desconoce $V_{n 1}$, y por tanto $r_{t}$.

A partir de (5) se obtienen las siguientes ecuaciones de transmisión del transformador:

$$
\left[\begin{array}{l}
\underline{V}_{1} \\
\underline{I}_{1}
\end{array}\right]=\left[\begin{array}{cc}
r_{t} & r_{t} \underline{Z}_{c c 2} \\
0 & 1 / r_{t}
\end{array}\right]\left[\begin{array}{l}
\underline{V}_{2} \\
\underline{I}_{2}
\end{array}\right]
$$

En estas ecuaciones, la relación de transformación $r_{t}$ será desconocida, aunque en realidad la incógnita sea la tensión nominal de primario $V_{n 1}$.

El objetivo es sintetizar un circuito pasivo a partir de estas ecuaciones de transmisión. La configuración del circuito será la que muestra la Figura 4.

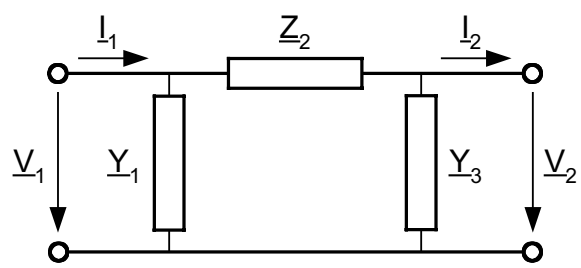

Figura 4. Circuito equivalente en $\pi$.

Las ecuaciones de transmisión de este circuito son las siguientes:

$\left[\begin{array}{l}\underline{V}_{1} \\ \underline{I}_{1}\end{array}\right]=\left[\begin{array}{cc}1+\underline{Z}_{2} \underline{Y}_{3} & \underline{Z}_{2} \\ \underline{Y}_{1}+\underline{Y}_{3}+\underline{Y}_{1} \underline{Y}_{3} \underline{Z}_{2} & 1+\underline{Z}_{2} \underline{Y}_{1}\end{array}\right]\left[\begin{array}{l}\underline{V}_{2} \\ \underline{I}_{2}\end{array}\right]$

De la comparación entre las ecuaciones (7) y (8) se deduce que:

$\underline{Z}_{2}=r_{t} \underline{Z}_{c c 2} ; 1+\underline{Z}_{2} \underline{Y}_{3}=r_{t} ; 1+\underline{Z}_{2} \underline{Y}_{1}=1 / r_{t}$

de donde se obtiene

$$
\begin{aligned}
& \underline{Z}_{2}=r_{t} \underline{Z}_{c c 2} \\
& \underline{Y}_{1}=\frac{1 / r_{t}-1}{\underline{Z}_{2}}=\frac{1-r_{t}}{r_{t}^{2} \underline{Z}_{c c 2}} \\
& \underline{Y}_{3}=\frac{r_{t}-1}{\underline{Z}_{2}}=\frac{r_{t}-1}{r_{t} \underline{Z}_{c c 2}}
\end{aligned}
$$

La Figura 5 muestra el circuito resultante, en el que $r_{t}$ suele ser una incógnita.

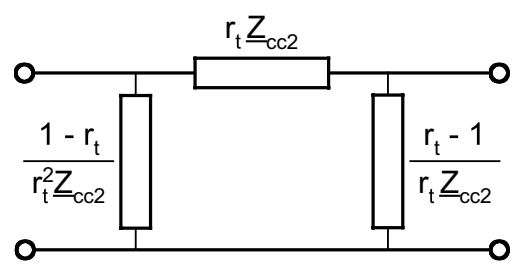

Figura 5. Circuito equivalente de un transformador visto desde el lado secundario.

La obtención del circuito equivalente con parámetros por unidad se puede realizar de varias formas. En este trabajo se realizará a partir de la ecuación (7), que puede expresarse en función de los valores base como sigue:

$$
\left[\begin{array}{cc}
V_{b 1} & 0 \\
0 & I_{b 1}
\end{array}\right]\left[\begin{array}{c}
\underline{v}_{1} \\
\underline{i}_{1}
\end{array}\right]=\left[\begin{array}{cc}
r_{t} & r_{t} \underline{Z}_{c c 2} \\
0 & 1 / r_{t}
\end{array}\right]\left[\begin{array}{cc}
V_{b 2} & 0 \\
0 & I_{b 2}
\end{array}\right]\left[\begin{array}{c}
\underline{v}_{2} \\
\underline{i}_{2}
\end{array}\right]
$$


donde $V_{b 1}, V_{b 2}, I_{b 1}$ y $I_{b 2}$ son los valores base de tensiones y corrientes, y $\underline{v}_{1}, \underline{v}_{2}, \underline{i}_{1}$ y $\underline{i}_{2}$ son los valores de tensiones y corrientes del transformador dados por unidad.

Nota: En este artículo los parámetros y variables (tensiones, corrientes y potencias) en valores por unidad se anotan con letras minúsculas, para diferenciarlos de los correspondientes valores expresados en cantidades físicas, que se anotan con letras mayúsculas.

La ecuación anterior queda de la siguiente forma:

$$
\left[\begin{array}{l}
\underline{v}_{1} \\
\underline{i}_{1}
\end{array}\right]=\left[\begin{array}{cc}
V_{b 1} & 0 \\
0 & I_{b 1}
\end{array}\right]^{-1}\left[\begin{array}{cc}
r_{t} & r_{t} \underline{Z}_{c c 2} \\
0 & 1 / r_{t}
\end{array}\right]\left[\begin{array}{cc}
V_{b 2} & 0 \\
0 & I_{b 2}
\end{array}\right]\left[\begin{array}{c}
\underline{v}_{2} \\
\underline{i}_{2}
\end{array}\right]
$$

de donde resulta:

$$
\left[\begin{array}{l}
\underline{v}_{1} \\
\underline{i}_{1}
\end{array}\right]=\left[\begin{array}{cc}
r_{t} / r_{b} & r_{t} \underline{Z}_{c c 2} I_{b 2} / V_{b 1} \\
0 & r_{b} / r_{t}
\end{array}\right]\left[\begin{array}{c}
\underline{v}_{2} \\
\underline{i}_{2}
\end{array}\right]
$$

siendo,

$$
r_{b}=\frac{V_{b 1}}{V_{b 2}}=\frac{I_{b 2}}{I_{b 1}}
$$

Teniendo en cuenta la expresión de $Z_{c c 2}$, ver ecuación (6), el término $r_{t} Z_{c c 2} I_{b 2} / V_{b 1}$ se puede escribir de la siguiente forma (nótese que se ha prescindido del carácter complejo de $Z_{c c 2}$ ).

$$
r_{t} Z_{c c 2} \frac{I_{b 2}}{V_{b 1}}=r_{t} \frac{V_{b 2}}{V_{b 1}} \varepsilon_{c c} \frac{V_{n 2}^{2}}{V_{b 2}^{2}} \frac{S_{b}}{S_{n}}
$$

de donde resulta:

$$
r_{t} Z_{c c 2} \frac{I_{b 2}}{V_{b 1}}=\frac{r_{t}}{r_{b}} \varepsilon_{c c}\left(\frac{V_{n 2}}{V_{b 2}}\right)^{2} \frac{S_{b}}{S_{n}}
$$

Puesto que el valor de la impedancia de cortocircuito por unidad vista desde el lado secundario es:

$$
z_{c c 2}=\frac{Z_{c c 2}}{Z_{b 2}}=\varepsilon_{c c}\left(\frac{V_{n 2}}{V_{b 2}}\right)^{2} \frac{S_{b}}{S_{n}}
$$

resulta el siguiente sistema de ecuaciones con variables y parámetros por unidad:

$$
\left[\begin{array}{l}
\underline{v}_{1} \\
\underline{i}_{1}
\end{array}\right]=\left[\begin{array}{cc}
r_{t} / r_{b} & \underline{z}_{c c 2} \cdot r_{t} / r_{b} \\
0 & r_{b} / r_{t}
\end{array}\right]\left[\begin{array}{l}
\underline{v}_{2} \\
\underline{i}_{2}
\end{array}\right]
$$

Haciendo,

$$
a=r_{t} / r_{b}
$$

queda finalmente:

$$
\left[\begin{array}{c}
\underline{v}_{1} \\
\underline{i}_{1}
\end{array}\right]=\left[\begin{array}{cc}
a & a \underline{z}_{c c 2} \\
0 & 1 / a
\end{array}\right]\left[\begin{array}{l}
\underline{v}_{2} \\
\underline{i}_{2}
\end{array}\right]
$$

Siguiendo el mismo procedimiento que con los circuitos anteriores, el circuito al que corresponden estas ecuaciones puede ser cualquiera de los que muestra la Figura 6.

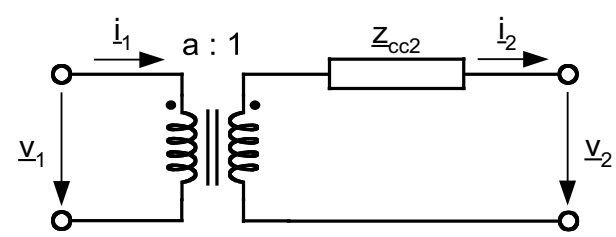

a) Con transformador ideal.

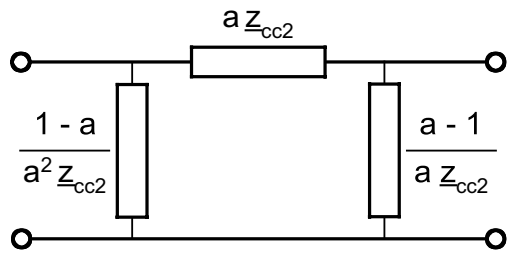

b) Sin transformador ideal.

Figura 6. Circuito equivalente de un transformador con parámetros en pu visto desde el lado secundario.

El circuito de la Figura 6 b es el que se suele analizar en la mayoría de textos cuando el objetivo del estudio es determinar la toma con la que debe operar un transformador para conseguir una determinada tensión en un nudo de la red en estudio.

\section{Impedancia de cortocircuito vista desde el lado primario}

La Figura 7 muestra el circuito equivalente de un transformador con regulación visto desde su lado primario. 


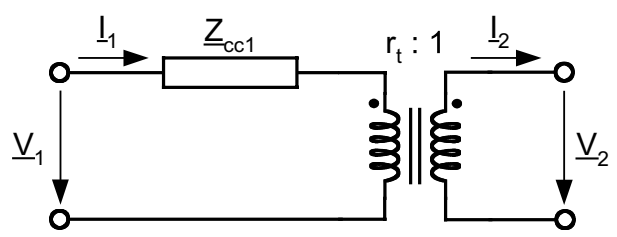

Figura 7. Circuito equivalente de un transformador visto desde el lado primario.

Las ecuaciones del circuito equivalente se pueden anotar de la siguiente forma:

$$
\begin{aligned}
& \underline{V}_{1}=r_{t} \underline{V}_{2}+\underline{Z}_{c c 1} \underline{I}_{1}=r_{t} \underline{V}_{2}+\underline{Z}_{c c 1} \underline{I}_{2} / r_{t} \\
& \underline{I}_{1}=\underline{I}_{2} / r_{t}
\end{aligned}
$$

donde:

$$
Z_{c c 1}=\varepsilon_{c c} \frac{V_{n 1}^{2}}{S_{n}}
$$

A partir de (21) se obtienen las siguientes ecuaciones de transmisión:

$$
\left[\begin{array}{c}
\underline{V}_{1} \\
\underline{I}_{1}
\end{array}\right]=\left[\begin{array}{cc}
r_{t} & \underline{Z}_{c c 1} / r_{t} \\
0 & 1 / r_{t}
\end{array}\right]\left[\begin{array}{l}
\underline{V}_{2} \\
\underline{I}_{2}
\end{array}\right]
$$

Siguiendo el mismo procedimiento que con el circuito anterior se obtiene el circuito de la Figura 8, en el que nuevamente la relación de transformación $r_{t}$ será una incógnita. En este caso la incógnita realmente es $V_{n 2}$, ya que $V_{n 1}$ es conocida y se utiliza para obtener $Z_{c c 1}$.

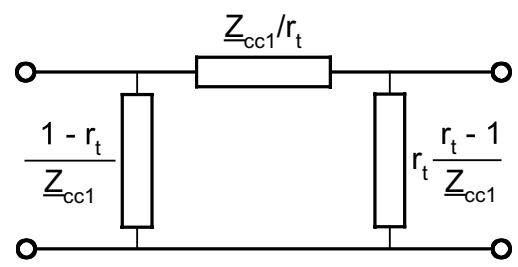

Figura 8. Circuito equivalente de un transformador visto desde el lado primario.

Las ecuaciones resultantes del circuito con parámetros por unidad son las siguientes:

$$
\left[\begin{array}{l}
\underline{v}_{1} \\
\underline{i}_{1}
\end{array}\right]=\left[\begin{array}{cc}
a & \underline{z}_{c c 1} / a \\
0 & 1 / a
\end{array}\right]\left[\begin{array}{l}
\underline{v}_{2} \\
\underline{i}_{2}
\end{array}\right]
$$

con

$$
z_{c c 1}=\frac{Z_{c c 1}}{Z_{b 1}}=\varepsilon_{c c}\left(\frac{V_{n 1}}{V_{b 1}}\right)^{2} \frac{S_{b}}{S_{n}}
$$

El circuito al que corresponden estas ecuaciones puede ser cualquiera de los que muestra la Figura 9 si se sigue el mismo procedimiento que se empleó cuando los parámetros eran reales.

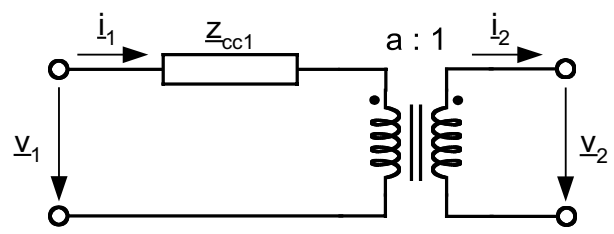

a) Con transformador ideal.

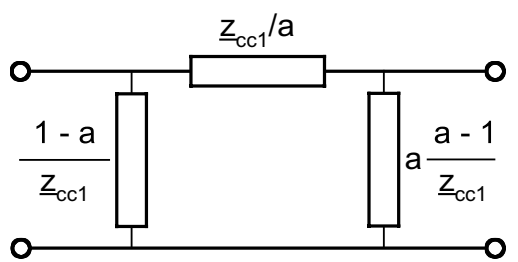

b) Sin transformador ideal.

Figura 9. Circuito equivalente de un transformador con parámetros en pu visto desde el lado primario.

El circuito de la Figura $9 \mathrm{~b}$ es utilizado en muy pocos textos, ver por ejemplo [6].

Se puede demostrar que los parámetros del circuito de la Figura 6b y los del circuito de la Figura 9b son los mismos cuando el valor de $a$ es conocido. Por ejemplo, para el parámetro central de ambos circuitos se tiene:

- Circuito equivalente Figura $6 \mathrm{~b}$

$$
a z_{c c 2}=\frac{r_{t}}{r_{b}} \frac{Z_{c c 2}}{Z_{b 2}}=\varepsilon_{c c} \frac{V_{n 1}}{V_{b 1}} \frac{V_{n 2}}{V_{b 2}} \frac{S_{b}}{S_{n}}
$$

- Circuito equivalente Figura 9b

$$
z_{c c 1} / a=\frac{r_{b}}{r_{t}} \frac{Z_{c c 1}}{Z_{b 1}}=\varepsilon_{c c} \frac{V_{n 1}}{V_{b 1}} \frac{V_{n 2}}{V_{b 2}} \frac{S_{b}}{S_{n}}
$$


Es decir, cuando los valores base y los valores nominales son conocidos, independientemente del valor de $a$, el estudio se puede hacer con uno u otro circuito, ver Ejemplo 2. Sin embargo, esto no significa que se pueda utilizar uno u otro circuito indistintamente cuando se trata de calcular el valor de $a$, como se muestra en el Ejemplo 1. El circuito de la Figura $6 \mathrm{~b}$ es el que debe utilizarse cuando se conoce la tensión nominal de secundario, y el de la Figura $9 \mathrm{~b}$ cuando se conoce la tensión nominal de primario.

\section{Otros circuitos equivalentes}

Los circuitos anteriores fueron obtenidos con valores base escogidos de forma arbitraria. Sin embargo, si la selección de los valores base se realiza siguiendo determinados criterios es posible obtener otros circuitos equivalentes, según se indica a continuación.

1. Si $r_{t}=r_{b}$, resulta $a=1$, y las ecuaciones (20) y (24) quedan, respectivamente:

$$
\begin{aligned}
& {\left[\begin{array}{l}
\underline{v}_{1} \\
\underline{i}_{1}
\end{array}\right]=\left[\begin{array}{cc}
1 & \underline{z}_{c c 2} \\
0 & 1
\end{array}\right]\left[\begin{array}{l}
\underline{v}_{2} \\
\underline{i}_{2}
\end{array}\right]} \\
& {\left[\begin{array}{l}
\underline{v}_{1} \\
\underline{i}_{1}
\end{array}\right]=\left[\begin{array}{cc}
1 & \underline{z}_{c c 1} \\
0 & 1
\end{array}\right]\left[\begin{array}{l}
\underline{v}_{2} \\
\underline{i}_{2}
\end{array}\right]}
\end{aligned}
$$

Puesto que se ha supuesto que:

$$
\frac{V_{n 1}}{V_{n 2}}=\frac{V_{b 1}}{V_{b 2}}
$$

también se cumple:

$$
\frac{V_{n 1}}{V_{b 1}}=\frac{V_{n 2}}{V_{b 2}}
$$

con lo que a partir de (17) y (25), o (27), resulta

$$
z_{c c 1}=z_{c c 2}=z_{c c}
$$

y el circuito resultante de un transformador con parámetros por unidad, visto tanto desde el lado primario como desde el lado secundario, será el que muestra la Figura 10.
Para obtener este circuito equivalente no es necesario que las tensiones base seleccionadas sean iguales a las tensiones nominales del transformador, sino que la relación entre tensiones base y tensiones nominales sea la misma (es decir, $r_{t}=r_{b} ; a=1$ ).

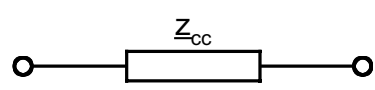

Figura 10. Circuito equivalente de un transformador con parámetros en $\mathrm{pu}-r_{t}=r_{b} ; a=1$.

2. En el caso particular en el que $V_{b 1}=V_{n 1} \mathrm{y}$ $V_{b 2}=V_{n 2}$, también es $a=1 \mathrm{y}$

$$
z_{c c 1}=z_{c c 2}=z_{c c}=\varepsilon_{c c} \frac{S_{b}}{S_{n}}
$$

3. Si además de la condición anterior se tiene $S_{b}=S_{n}$, entonces:

$$
z_{c c 1}=z_{c c 2}=z_{c c}=\varepsilon_{c c}
$$

Aunque la relación de transformación, $r_{t}=V_{n 1} / V_{n 2}$, no sea conocida, es posible suponer $a=1$ como se muestra en el siguiente ejemplo. Sin embargo, no siempre resulta aconsejable hacer $r_{t}=r_{b}$; en tales casos se tendrá que escoger, entre el circuito de la Figura 5 o el de la Figura 8, y utilizar $a\left(=r_{t} / r_{b} \neq 1\right)$ como incógnita.

\section{EJEMPLOS}

Los circuitos equivalentes del transformador con regulación se obtuvieron para un transformador monofásico. Tanto el procedimiento como los circuitos equivalentes y el cálculo de parámetros serán iguales en caso de tratar con transformadores trifásicos. En el caso de transformadores trifásicos, la potencia nominal, $S_{n}$, es la correspondiente a las tres fases, mientras que las tensiones nominales, $V_{n 1}$ y $V_{n 2}$, son las tensiones eficaces (rms) entre fases. Los transformadores estudiados en los ejemplos que se presentan a continuación son trifásicos.

Un objetivo común a los dos ejemplos incluidos en esta sección es mostrar cómo se pueden utilizar los distintos circuitos de un transformador con regulación analizados en la sección anterior. 


\section{Ejemplo 1.}

Este ejemplo incluye dos partes en las que se estudia el mismo transformador, aunque los valores a determinar serán distintos en cada parte. En cada caso se trata de determinar una de las tensiones nominales del transformador. El circuito empleado para representar el transformador dependerá de la tensión desconocida en cada caso.

Aunque el procedimiento es el mismo en ambas partes, existen algunas diferencias importantes que justifican el estudio de los dos casos. Téngase en cuenta que el transformador en estudio puede ser tanto un transformador con tomas (para el que se desea determinar la relación de transformación necesaria) como un transformador con relación de transformación fija (para el que se desea determinar una de sus tensiones nominales).

\section{A. Cálculo de la tensión nominal de primario}

Considérese el transformador mostrado en la Figura 11. Se trata de un transformador con relación de transformación variable, para el que se puede regular su tensión nominal de primario. Se desea determinar el valor de la tensión nominal de primario, $V_{n 1}$, para que atendiendo la demanda indicada en la figura, la tensión resultante en el lado secundario sea $25 \mathrm{kV}$ cuando la tensión en su lado primario es $220 \mathrm{kV}$.

El ejemplo se resolverá utilizando dos procedimientos distintos, y en ambos casos los cálculos se realizarán con cantidades por unidad. Como valor de potencia base se utilizará $S_{b}=100$ MVA.

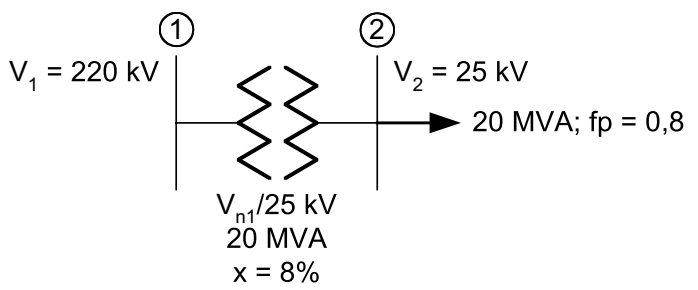

Figura 11.Ejemplo 1A: Diagrama del caso a estudiar.

\section{1) Parámetro $a=1$}

Los valores base para las tensiones serán los valores nominales, y el circuito equivalente será el que muestra la Figura 12.

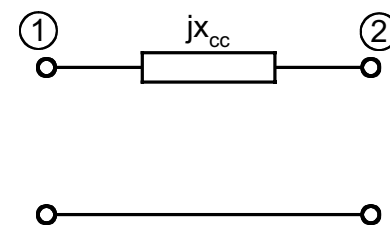

Figura 12.Ejemplo 1A: Circuito equivalente del transformador $(a=1)$.

Los valores base y los nominales son, por tanto:

- Tensiones: Nominales $=V_{n 1} / 25 \mathrm{kV}$

$$
\text { Base }=V_{n 1} / 25 \mathrm{kV}
$$

- Potencia: Nominal $=20 \mathrm{MVA}$

$$
\text { Base }=100 \text { MVA. }
$$

Obsérvese que tanto el valor base como el valor nominal de la tensión en el lado primario son desconocidos. En realidad se trata del valor a obtener en este ejemplo.

De acuerdo con la ecuación (17) se tiene:

$$
x_{c c}=x \cdot\left(\frac{V_{n 2}}{V_{b 2}}\right)^{2} \frac{S_{b}}{S_{n}}=0,08 \cdot\left(\frac{25}{25}\right)^{2} \cdot \frac{100}{20}=0,40
$$

Las condiciones de operación conocidas son las siguientes:

- Tensión nudo 1: $\quad V_{1}=220 \mathrm{kV}$

- Tensión nudo 2: $\quad V_{2}=25 \mathrm{kV}$

- Demanda en nudo 2: $S_{D 2}=20 \mathrm{MVA}, \mathrm{fp}=0,8$.

A partir de los valores base se obtienen los siguientes valores por unidad:

Tensión nudo 2: $\quad v_{2}=1 \mathrm{pu}$

Demanda en nudo 2: $\underline{s}_{D 2}=0,16+\mathrm{j} 0,12 \mathrm{pu}$.

El valor de la tensión en el nudo 1 se puede obtener mediante la siguiente expresión:

$$
\underline{v}_{1}=\underline{v}_{2}+j x_{c c} \underline{i}_{2}
$$

Haciendo $\underline{v}_{2}=1 \angle 0^{\circ}$, el valor de la intensidad $i_{2}$ será

$$
\underline{i}_{2}=\left(\frac{\underline{s}_{D 2}}{v_{2}}\right)^{*}=0,16-j 0,12 \mathrm{pu} \quad\left(\underline{s}_{D 2}=\underline{v}_{2} \underline{i}_{2}^{*}\right)
$$

de donde resulta:

$$
\underline{v}_{1}=1,048+j 0,064 \mathrm{pu} \quad \Rightarrow \quad\left|\underline{v}_{1}\right|=1,05 \mathrm{pu}
$$


Puesto que

$$
\left|\underline{v}_{1}\right|=\frac{V_{1}}{V_{b 1}}=\frac{V_{1}}{V_{n 1}}
$$

y $V_{1}=220 \mathrm{kV}$, se obtiene

$$
V_{n 1}=\frac{220}{1,05} \approx 209,5 \mathrm{kV}
$$

\section{2) Parámetro $a \neq 1$}

Los valores base para las tensiones serán los valores de las tensiones conocidas en ambos lados del transformador, y el circuito equivalente será el que muestra la Figura 13. Recuérdese que el parámetro central se expresa como impedancia y los parámetros transversales como admitancias.

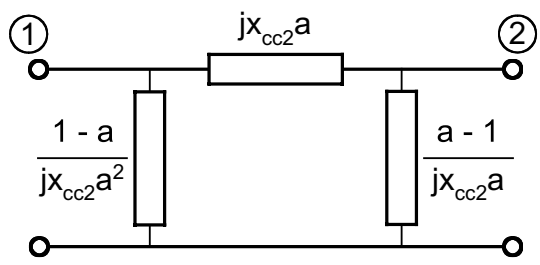

Figura 13. Ejemplo 1A: Circuito equivalente del transformador $(a \neq 1)$.

Los valores base y los nominales son ahora los siguientes:
- Tensiones:
Nominales $=V_{n 1} / 25 \mathrm{kV}$
- Potencia:$$
\text { Base }=220 / 25 \mathrm{kV}
$$
Nominal $=20 \mathrm{MVA}$
Base $=100$ MVA.

La reactancia de cortocircuito del transformador se obtiene de nuevo mediante la ecuación (17), con lo que se obtiene el mismo valor que se obtuvo anteriormente; es decir, $x_{c c 2}=0,40 \mathrm{pu}$.

Las condiciones de operación conocidas son las mismas que en el estudio anterior. Sin embargo, para las condiciones de operación conocidas por unidad hay una novedad, ya que ahora también se conoce la tensión en el nudo 1 :

- Tensión nudo 1: $\quad v_{1}=1 \mathrm{pu}$

- Tensión nudo 2: $\quad v_{2}=1 \mathrm{pu}$

- Demanda en nudo 2: $\underline{s}_{D 2}=0,16+j 0,12 \mathrm{pu}$.

Por lo que respecta al parámetro $a$ se tiene

$$
a=\frac{r_{t}}{r_{b}}=\frac{V_{n 1} / 25}{220 / 25}=\frac{V_{n 1}}{220}
$$

de donde resulta $V_{n 1}=220 a$.

El ejemplo se resolverá mediante el método de Newton-Raphson, siguiendo el mismo procedimiento que se emplea en la resolución del flujo de cargas (load flow) $[6,15,16]$. Para el tratamiento de transformadores trifásicos en estudios de flujo de cargas, ver [17-18].

De la Figura 13 se obtiene la siguiente matriz de admitancias de nudo:

$$
Y_{B U S}=\left[\begin{array}{cc}
\frac{1}{j x_{c c 2} a^{2}} & -\frac{1}{j x_{c c 2} a} \\
-\frac{1}{j x_{c c 2} a} & \frac{1}{j x_{c c 2}}
\end{array}\right]
$$

Teniendo en cuenta que $x_{c c 2}=0,4$ queda:

$$
Y_{B U S}=\left[\begin{array}{cc}
-j \frac{2,5}{a^{2}} & j \frac{2,5}{a} \\
j \frac{2,5}{a} & -j 2,5
\end{array}\right]
$$

Las ecuaciones de potencia en el nudo 2, a partir de las ecuaciones de admitancia de nudo del circuito de la Figura 13, son las siguientes:

$$
\begin{aligned}
& p_{2}=v_{2}\left[v_{1}\left(g_{21} \cos \delta_{21}+b_{21} \sin \delta_{21}\right)+v_{2} g_{22}\right] \\
& q_{2}=v_{2}\left[v_{1}\left(g_{21} \sin \delta_{21}-b_{21} \cos \delta_{21}\right)-v_{2} b_{22}\right]
\end{aligned}
$$

donde $v_{1}$ y $v_{2}$ son los módulos de las tensiones en los nudos terminales del transformador, en por unidad, $\delta_{21}=\delta_{2}-\delta_{1}, \delta_{1}$ y $\delta_{2}$ son los argumentos de las tensiones, y $g_{i j}$ y $b_{i j}$ son respectivamente la parte real e imaginaria de los elementos de la matriz de admitancias de nudo, en por unidad.

Teniendo en cuenta los valores de la matriz de admitancias de nudo queda:

$$
\begin{aligned}
& p_{2}=v_{2} v_{1} b_{21} \sin \delta_{21} \\
& q_{2}=-v_{2}\left[v_{1} b_{21} \cos \delta_{21}+v_{2} b_{22}\right]
\end{aligned}
$$


Si se toma el ángulo de fase de la tensión en el nudo 1 como ángulo de referencia, $\delta_{1}=0$, queda $\delta_{21}=\delta_{2}=\delta$. Puesto que:

$$
v_{1}=1 \quad v_{2}=1 \quad b_{21}=\frac{2,5}{a} \quad b_{22}=-2,5
$$

resulta finalmente,

$$
\begin{aligned}
& p_{2}=\frac{2,5}{a} \sin \delta \\
& q_{2}=-\frac{2,5}{a} \cos \delta+2,5
\end{aligned}
$$

Para la resolución mediante el método de NewtonRaphson se establecen las siguientes ecuaciones:

$$
\begin{aligned}
& f_{p}=p_{2 \text { conocida }}-p_{2 \text { calculada }}=0 \\
& f_{q}=q_{2 \text { conocida }}-q_{2 \text { calculada }}=0
\end{aligned}
$$

que en este caso quedan de la siguiente forma:

$$
\begin{aligned}
& f_{p}=-0,16-\frac{2,5}{a} \sin \delta=0 \\
& f_{q}=-2,62+\frac{2,5}{a} \cos \delta=0
\end{aligned}
$$

Se tiene un sistema de dos ecuaciones con dos incógnitas, $a$ y $\delta$. El método de Newton-Raphson se basará en el siguiente algoritmo:

$$
\left[\begin{array}{l}
\delta \\
a
\end{array}\right]_{(m+1)}=\left[\begin{array}{l}
\delta \\
a
\end{array}\right]_{(m)}-\left[\begin{array}{ll}
\partial f_{p} / \partial \delta & \partial f_{p} / \partial a \\
\partial f_{q} / \partial \delta & \partial f_{q} / \partial a
\end{array}\right]_{(m)}^{-1}\left[\begin{array}{l}
f_{p} \\
f_{q}
\end{array}\right]_{(m)}
$$

Si se inicia el proceso iterativo con $\delta_{(0)}=0 \mathrm{y}$ $a_{(0)}=1$, se obtiene $\delta=-3,495^{\circ} ; a=0,9524$. De aquí resulta $V_{n 1}=220 \mathrm{a}=220 \cdot 0,9524 \approx 209,5 \mathrm{kV}$.

\section{B. Cálculo de la tensión nominal de secundario} Considérese ahora el transformador mostrado en la Figura 14. Se trata del mismo transformador anterior pero para el que ahora se regula su tensión nominal de secundario. Se desea determinar el valor de esta tensión nominal, $V_{n 2}$, para que atendiendo la demanda indicada en la figura, la tensión resultante en el lado secundario sea $25 \mathrm{kV}$ cuando la tensión en su lado primario es $220 \mathrm{kV}$.

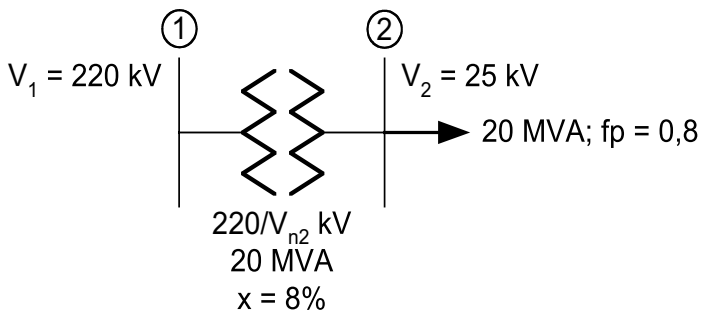

Figura 14.Ejemplo 1B: Diagrama del caso a estudiar.

\section{1) Parámetro $a=1$}

Los valores base para las tensiones serán los valores nominales, y el circuito equivalente será el mismo que muestra la Figura 12. Es decir:

$$
\begin{array}{ccl}
\text { - } & \text { Tensiones: } & \text { Nominales }=220 / V_{n 2} \mathrm{kV} \\
& & \text { Base }=220 / V_{n 2} \mathrm{kV} \\
\text { - } & \text { Potencia: } & \text { Nominal }=20 \mathrm{MVA} \\
& & \text { Base }=100 \mathrm{MVA} .
\end{array}
$$

Siguiendo el mismo procedimiento que en el ejemplo anterior, de acuerdo con la ecuación (25) se obtiene el mismo valor, $x_{c c}=0,40$.

Las condiciones de operación son las mismas que en el caso anterior. A partir de los valores base se obtienen los siguientes valores por unidad:

- Tensión nudo 1: $\quad v_{1}=1 \mathrm{pu}$

- Demanda en nudo 2: $\underline{s}_{D 2}=0,16+j 0,12 \mathrm{pu}$.

El valor de la tensión en el nudo 2 se puede obtener resolviendo la siguiente ecuación:

$$
v_{2}^{4}+\left(2\left(r_{c c} p_{D 2}+x_{c c} q_{D 2}\right)-v_{1}^{2}\right) v_{2}^{2}+\left(z_{c c} s_{D 2}\right)^{2}=0
$$

en la que

$$
\begin{array}{llll}
v_{1}=1 & s_{D 2}=0,20 & p_{D 2}=0,16 & q_{D 2}=0,12 \\
z_{c c}=0,4 & r_{c c}=0 & x_{c c}=0,4 &
\end{array}
$$

Nota: La deducción de la ecuación (34) se presenta en el Anexo de este artículo.

Resulta $v_{2}=0,9470$. Puesto que:

$$
\left|\underline{v}_{2}\right|=\frac{V_{2}}{V_{b 2}}=\frac{V_{2}}{V_{n 2}}
$$


y $V_{2}=25 \mathrm{kV}$, se obtiene:

$$
V_{n 2}=\frac{25}{0,9470} \approx 26,4 \mathrm{kV}
$$

\section{2) Parámetro $a \neq 1$}

Los valores base para las tensiones serán los valores de las tensiones conocidas en ambos lados del transformador, y el circuito equivalente será el que muestra la Figura 15.

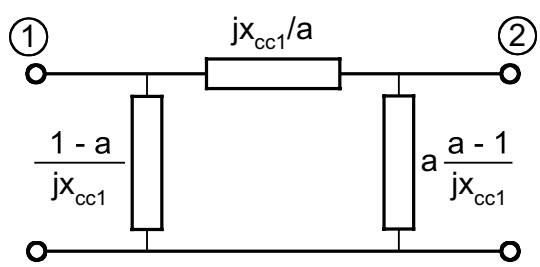

Figura 15. Ejemplo 1B: Circuito equivalente del transformador $(a \neq 1)$.

Los valores base y los valores nominales son ahora los siguientes:

- Tensiones: Nominales $=220 / V_{n 2} \mathrm{kV}$

Base $=220 / 25 \mathrm{kV}$

- Potencia: Nominal $=20 \mathrm{MVA}$

Base $=100$ MVA.

La reactancia de cortocircuito del transformador se obtiene de nuevo mediante la ecuación (25), que proporciona el mismo valor que se obtuvo en el Ejemplo 1A; es decir, $x_{c c 1}=0,40 \mathrm{pu}$.

Las condiciones de operación conocidas son las mismas que en el estudio anterior. Sin embargo, para las condiciones de operación por unidad ahora también se conoce la tensión en el nudo 2 :

- Tensión nudo 1: $v_{1}=1 \mathrm{pu}$

- Tensión nudo 2: $v_{2}=1 \mathrm{pu}$

- Demanda en 2: $\underline{s}_{D 2}=0,16+\mathrm{j} 0,12 \mathrm{pu}$.

Por lo que respecta al parámetro $a$ se tiene

$$
a=\frac{r_{t}}{r_{b}}=\frac{220 / V_{n 2}}{220 / 25}=\frac{25}{V_{n 2}}
$$

de donde resulta $V_{n 2}=25 / a$.

De la Figura 15 se obtiene la siguiente matriz de admitancias de nudo:

$$
Y_{B U S}=\left[\begin{array}{cc}
\frac{1}{j x_{c c 1}} & -\frac{a}{j x_{c c 1}} \\
-\frac{a}{j x_{c c 1}} & \frac{a^{2}}{j x_{c c 1}}
\end{array}\right]
$$

Teniendo en cuenta que $x_{c c 1}=0,4$, queda

$$
Y_{B U S}=\left[\begin{array}{cc}
-j 2,5 & j 2,5 a \\
j 2,5 a & -j 2,5 a^{2}
\end{array}\right]
$$

Las ecuaciones de potencia en el nudo 2, a partir de las ecuaciones de admitancia de nudo del circuito de la Figura 15 son las mismas que para el ejemplo anterior. Si se toma de nuevo el ángulo de fase de la tensión en el nudo 1 como ángulo de referencia, $\delta_{1}=0$, y se tiene en cuenta que:

$$
v_{1}=1 \quad v_{2}=1 \quad b_{21}=2,5 a \quad b_{22}=-2,5 a^{2}
$$

resulta finalmente:

$$
\begin{aligned}
& p_{2}=2,5 a \sin \delta \\
& q_{2}=-2,5 a \cos \delta+2,5 a^{2}
\end{aligned}
$$

Con el mismo procedimiento que en el caso anterior se obtiene el siguiente sistema de ecuaciones:

$$
\begin{aligned}
& f_{p}=-0,16-2,5 a \sin \delta=0 \\
& f_{q}=-0,12+2,5 a \cos \delta-2,5 a^{2}=0
\end{aligned}
$$

La aplicación del método de Newton-Raphson se basará en el mismo algoritmo que en el Ejemplo 1A. Se obtiene $\delta=-3,875^{\circ} ; a=0,947$. De aquí resulta $V_{n 1} \approx 26,4 \mathrm{kV}$.

Del estudio de las situaciones anteriores, con $a \neq 1$, se obtiene una conclusión interesante. En ambos casos se conocen las mismas condiciones en valores por unidad, pero los sistemas de ecuaciones a resolver, (33) y (35), son distintos, y los resultados son diferentes. El uso de uno u otro circuito viene impuesto por el valor de tensión nominal que se ha de obtener: en el Ejemplo 1A se tenía que determinar $V_{n 1}$, mientras que en el Ejemplo $1 \mathrm{~B}$ ha sido $V_{n 2}$. 


\section{Ejemplo 2}

La Figura 16 muestra un transformador conectado a una red de media tensión que alimenta una instalación industrial. Se desea calcular la corriente de cortocircuito, medida en su lado secundario.

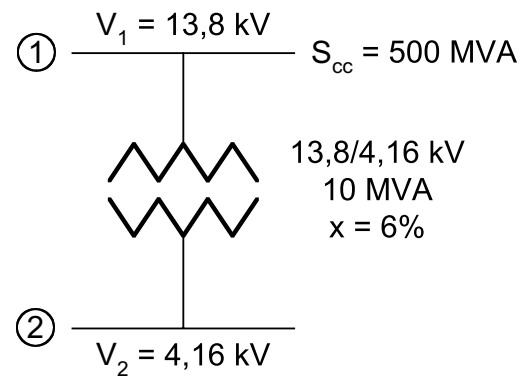

Figura 16.Ejemplo 2: Diagrama del caso a estudiar.

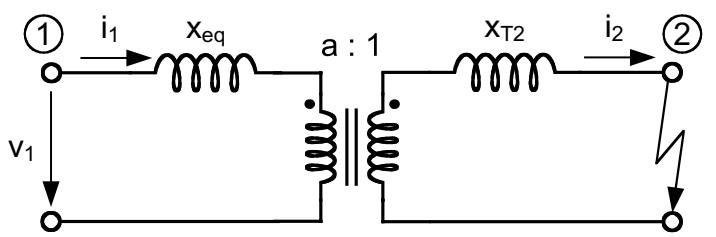

a) Impedancia de cortocircuito en secundario.

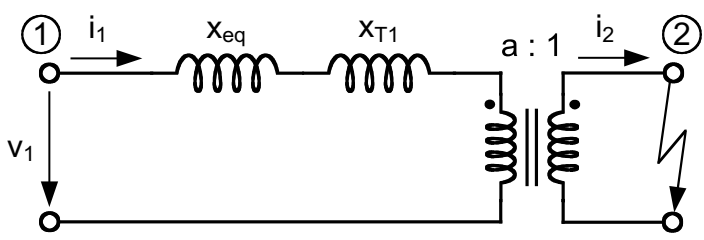

b) Impedancia de cortocircuito en primario.

Figura 17. Ejemplo 2: Circuitos equivalentes.

El objetivo es calcular la corriente de cortocircuito considerando varios escenarios en los que se varía la toma del transformador y las condiciones de operación. Los cálculos serán realizados con cantidades por unidad, tomando como referencia los circuitos de la Figura 17, en los que $x_{e q}$ representa la impedancia de la red de $13,8 \mathrm{kV}$. El caso ha sido propuesto en [19] y aquí será analizado considerando los modelos y procedimientos presentados en las secciones anteriores.

Teniendo en cuenta que todos los cálculos se realizarán con los mismos valores base $\left(S_{b}=10\right.$ MVA, $\left.V_{b 1}=13,8 \mathrm{kV}, V_{b 2}=4,16 \mathrm{kV}\right)$, el cálculo de la corriente de cortocircuito, medida en los terminales del lado secundario, se obtiene a partir de los dos circuitos como sigue:
- Circuito equivalente visto desde el lado secundario

$$
\begin{gathered}
v_{1}=x_{e q} \frac{i_{c c 2}}{a}+a x_{T 2} i_{c c 2}=\left(\frac{x_{e q}}{a}+a x_{T 2}\right) i_{c c 2} \\
i_{c c 2}=\frac{v_{1}}{\left(\frac{x_{e q}}{a}+a x_{T 2}\right)}=a \frac{v_{1}}{x_{e q}+a^{2} x_{T 2}}
\end{gathered}
$$

Puesto que $S_{b}=S_{n}$, y

$$
a=\frac{r_{t}}{r_{b}}=\frac{V_{n 1}}{V_{n 2}} \frac{V_{b 2}}{V_{b 1}} \quad v_{1}=\frac{V_{1}}{V_{b 1}}
$$

$$
\begin{gathered}
I_{c c 2}=i_{c c 2} I_{b 2} \quad I_{b 2}=\frac{S_{b}}{\sqrt{3} V_{b 2}}=\frac{S_{n}}{\sqrt{3} V_{b 2}} \\
x_{T 2}=x \cdot\left(\frac{V_{n 2}}{V_{b 2}}\right)^{2} \frac{S_{b}}{S_{n}}=x \cdot\left(\frac{V_{n 2}}{V_{b 2}}\right)^{2} \\
x_{e q}=\frac{S_{b}}{S_{c c}}=\frac{S_{n}}{S_{c c}}
\end{gathered}
$$

resulta:

$$
I_{c c 2}=\frac{V_{1}}{V_{b 1}} \frac{V_{n 1}}{V_{b 1}} \frac{S_{n}}{\sqrt{3} V_{n 2}\left(x_{e q}+x \cdot\left(\frac{V_{n 1}}{V_{b 1}}\right)^{2}\right)}
$$

- Circuito equivalente visto desde el lado primario

$$
v_{1}=\left(x_{e q}+x_{T 1}\right) \frac{i_{c c 2}}{a} \quad i_{c c 2}=a \frac{v_{1}}{x_{e q}+x_{T 1}}
$$

Puesto que

$$
x_{T 1}=x \cdot\left(\frac{V_{n 1}}{V_{b 1}}\right)^{2} \frac{S_{b}}{S_{n}}=x \cdot\left(\frac{V_{n 1}}{V_{b 1}}\right)^{2}
$$

resulta la misma expresión que se obtuvo con el circuito visto desde el lado secundario. 
Para el caso base, ver Figura 16, se tienen los siguientes valores: $S_{n}=S_{b}=10 \mathrm{MVA}, V_{n 1}=V_{b 1}=13,8$ $\mathrm{kV}, V_{n 2}=V_{b 2}=4,16 \mathrm{kV}, x=0,06, V_{1}=13,8 \mathrm{kV}$, $x_{e q}=0,02$, de donde resulta $I_{c c 2}=17,53 \mathrm{KA}$.

La Tabla 1 muestra los resultados obtenidos a partir de la expresión anterior con algunos de los casos analizados en la referencia [19]. Se puede comprobar que en todos los casos, los resultados son los mismos que se obtuvieron en [19].

Este ejemplo demuestra que la aplicación de uno u otro circuito produce los mismos resultados cuando el valor de $a$ es conocido.

Tabla 1. Ejemplo 2: Resultados en función de la toma de regulación y de las condiciones de operación.

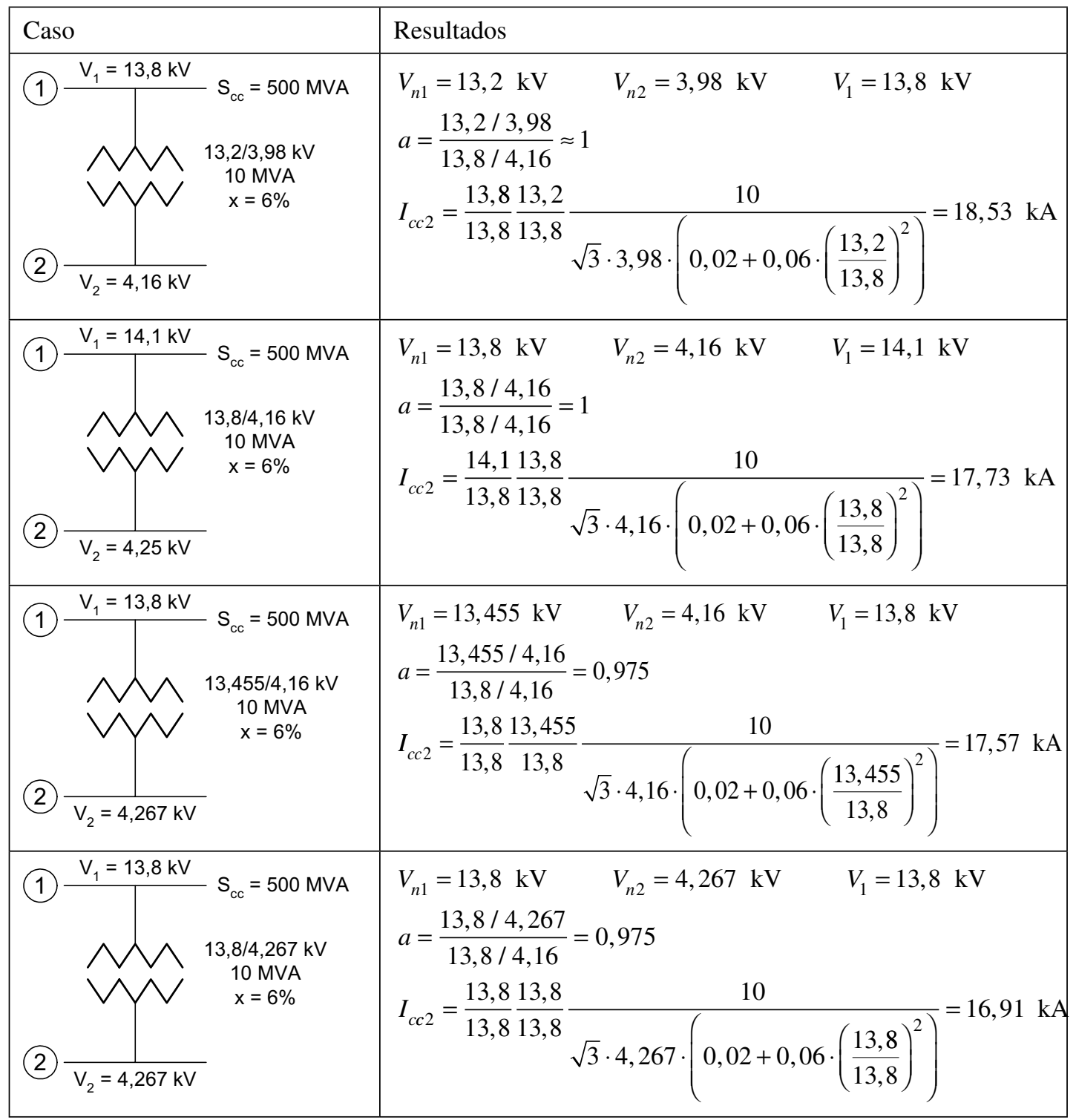




\begin{tabular}{|l|l|}
\hline Caso & Resultados \\
\hline (1) $\mathrm{V}_{1}=13,54 \mathrm{kV}$ \\
(2)
\end{tabular}

Nota: En todos los cálculos $V_{b 1}=13,8 \mathrm{kV} ; V_{b 2}=4,16 \mathrm{kV} ; S_{n}=10$ MVA.

\section{CONCLUSIONES}

Este artículo ha mostrado cómo determinar el valor de la tensión nominal con la que debe trabajar un transformador con regulación que forma parte de una red en la que se controla la tensión en alguno de sus nudos mediante una relación de transformación adecuada. Por otra parte, se ha mostrado que es posible obtener más de un circuito equivalente para un transformador con regulación, y que el empleo de uno u otro circuito dependerá de los valores conocidos, del estudio a realizar, y del método de solución aplicado. Se ha demostrado que en algunos casos es posible utilizar circuitos muy simples.

Las conclusiones más importantes se pueden resumir de la siguiente forma:

1. Los cálculos en un transformador con valores por unidad se pueden realizar seleccionando los valores base $\left(S_{b}, V_{b 1}, V_{b 2}\right)$ de forma arbitraria.

2. En el caso más general (por ejemplo, cuando los valores de tensiones base son seleccionados de forma arbitraria), el cálculo de los parámetros del circuito equivalente de un transformador con relación de transformación variable será diferente visto desde el lado primario que visto desde el lado secundario. Téngase en cuenta que el parámetro $a$ es un valor desconocido si se desconoce una de las tensiones nominales del transformador.

3. Si la selección de valores base se hace suponiendo que $r_{b}=r_{t}$, entonces el circuito equivalente resulta más simple y los parámetros son iguales vistos desde cualquiera de los lados del transformador. Se ha mostrado, por otro lado, que en algunos ejemplos muy simples, en los que es posible hacer los cálculos de forma manual, se puede suponer $a=1$ aunque no se conozcan las dos tensiones nominales del transformador.

4. No siempre es posible o aconsejable escoger $r_{b}=r_{t}$. Por ejemplo, cuando se desea obtener la relación de transformación mediante técnicas de flujo de cargas, lo que puede ser necesario en caso de que el transformador forme parte de una red o sistema grande. En tal caso es importante tener en cuenta que el circuito equivalente a utilizar en los cálculos cuando $r_{b} \neq r_{t}$, y $a \neq 1$, se debe escoger entre el circuito de la Figura $6 \mathrm{~b}$ o el de la Figura $9 \mathrm{~b}$.

\section{REFERENCIAS}

[1] M.J. Heathcote. "The J \& P Transformer Book". Newnes. Twelfth Edition. 1998.

[2] R.M. del Vecchio, B. Poulin, P.T. Feghali, D.M. Shah and R. Ahuja. "Transformer Design Principles". CRC Press. Second Edition. 2010.

[3] J.H. Harlow (Ed.). "Electric Power Transformer Engineering”. CRC Press. 2004.

[4] Bharat Heavy Electricals Ltd. "Transformers". McGraw-Hill. 2005.

[5] S.V. Kulkarni and S.A. Khaparde. "Transformer Engineering Design and Practice". Marcel Dekker, Inc. 2004.

[6] J.J. Grainger and W.D. Stevenson. "Power System Analysis". McGraw-Hill. 1994.

[7] O.I. Elgerd. "Electric Energy Systems Theory. An Introduction". McGraw-Hill. Second Edition. 1982.

[8] CIGRE Working Group 02 (SC 33). "Guidelines for Representation of Network 
Elements when Calculating Transients". CIGRE Technical Brochure. No 39. 1990.

[9] A.M. Gole, J.A. Martinez-Velasco and A.J.F. Keri (Eds.). "Modeling and Analysis of System Transients Using Digital Programs". IEEE PES Special Publication. TP-133-0. 1999.

[10] J.A. Martinez and B.A. Mork. "Transformer modeling for low- and mid-frequency transients - A review". IEEE Trans. on Power Delivery. Vol. 20, Issue 2, pp. 1625-1632. Abril, 2005.

[11] J.A. Martinez, R. Walling, B.A. Mork, J. Martin-Arnedo and D. Durbak. "Parameter determination for modeling system transients - Part III: Transformers". IEEE Trans. on Power Delivery. Vol. 20, Issue 3, pp. 20512062. July, 2005.

[12] F. de León, P. Gómez, J.A. Martinez-Velasco and M. Rioual. "Transformers". In: J.A. Martinez-Velasco (Ed.). Power System Transients. Parameter Determination. CRC Press. 2009.

[13] J. Duncan Glover, M. Sarma and T. Oerbye. "Power System Analysis and Design". CL Engineering. Fourth Edition. 2009.

\section{ANEXO}

En este anexo se deducirá la ecuación (34), empleada en la segunda parte del Ejemplo 1. Se supone que el circuito equivalente del transformador a estudiar es el que se muestra en la Figura A1. Las condiciones de operación conocidas incluyen la tensión en el primario del transformador, $V_{1}$, y la demanda de potencia en el secundario, $S_{D 2}$.

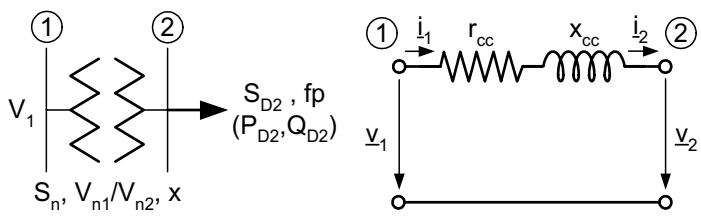

Figura A1. Diagrama y circuito equivalente del caso a estudiar.

La Figura A1 muestra las variables y parámetros a considerar en el estudio. Se supone que todos los valores del circuito equivalente están expresados por unidad. La ecuación del circuito es la siguiente:

$$
\underline{v}_{1}=\underline{v}_{2}+\underline{z}_{c c} \underline{i}_{2}
$$

[14] A.R. Bergen and V.J. Vittal. "Power System Analysis". Prentice Hall. Second Edition. 2000.

[15] N.M. Peterson and W. Scott Meyer. "Automatic adjustment of transformer and phase-shifter taps in the Newton power flow". IEEE Trans. on Power Apparatus and Systems. Vol. 90, Issue 1, pp. 103-108. January - February, 1971.

[16] J.C. Das. "Power system analysis: shortcircuit load flow and harmonics". Marcel Dekker, Inc. 2002.

[17] N.N. Bengiamin. "Regulating transformer model for use in load flow analysis". IEEE Trans. on Power Apparatus and Systems. Vol. 104, Issue 5, pp. 1102-1108. 1985.

[18] P.A.N. Garcia, J.L.R. Pereira and S. Carneiro. "Voltage control devices models for distribution power flow analysis". IEEE Transactions on Power Systems. Vol. 16, Issue 4, pp. 586-594. November, 2001.

[19] IEEE Std 551-2006. Violet Book. "IEEE Recommended Practice for Calculating Short-Circuit Currents in Industrial and Commercial Power Systems”. 2006.

Por otra parte

$$
\underline{s}_{D 2}=\underline{v}_{2} \underline{i}_{2}^{*}
$$

De (A2) se obtiene

$$
\underline{i}_{2}=\left(\frac{\underline{s}_{D 2}}{\underline{v}_{2}}\right)^{*}
$$

que sustituida en (A1) da

$$
\underline{v}_{1}=\underline{v}_{2}+\underline{z}_{c c}\left(\frac{\underline{s}_{D 2}}{\underline{v_{2}}}\right)^{*}
$$

Si se hace:

$$
\begin{gathered}
\underline{v}_{2}=v_{2 \angle 0^{\circ}} \quad \underline{v}_{1}=v_{1 \angle \delta} \\
\underline{z}_{c c}=r_{c c}+j x_{c c}
\end{gathered}
$$




$$
\underline{s}_{D 2}=p_{D 2}+j q_{D 2}
$$

y se separan la parte real y la parte imaginaria del lado derecho de la ecuación (A3) resulta:

$\underline{v}_{1}=\left(v_{2}+\frac{r_{c c} p_{D 2}+x_{c c} q_{D 2}}{v_{2}}\right)+j\left(\frac{x_{c c} p_{D 2}-r_{c c} q_{D 2}}{v_{2}}\right)$

Si se multiplica cada lado de esta ecuación por su conjugado complejo queda:

$$
v_{1}^{2}=\left(v_{2}+\frac{r_{c c} p_{D 2}+x_{c c} q_{D 2}}{v_{2}}\right)^{2}+\left(\frac{x_{c c} p_{D 2}-r_{c c} q_{D 2}}{v_{2}}\right)^{2}
$$

En esta ecuación sólo hay un valor desconocido, $v_{2}$. $\mathrm{Si}$ se multiplican ambos lados por $v_{2}^{2}$, se obtiene:

$v_{1}^{2} v_{2}^{2}=\left(v_{2}^{2}+\left(r_{c c} p_{D 2}+x_{c c} q_{D 2}\right)\right)^{2}+\left(x_{c c} p_{D 2}-r_{c c} q_{D 2}\right)^{2}$

Desarrollando los dos binomios resulta finalmente:

$v_{2}^{4}+\left(2\left(r_{c c} p_{D 2}+x_{c c} q_{D 2}\right)-v_{1}^{2}\right) v_{2}^{2}+\left(z_{c c} s_{D 2}\right)^{2}=0(\mathrm{~A} 5)$

La ecuación tiene cuatro soluciones, sólo dos de las cuales son positivas y por tanto aceptables, ya que se trata de obtener el módulo de la tensión en el nudo 2. Las otras dos soluciones son negativas y de valor absoluto igual a las soluciones con signo positivo. 